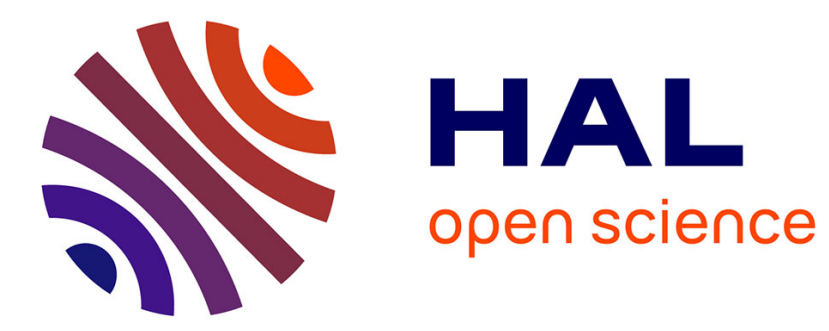

\title{
Transition from non-swirling to swirling axisymmetric turbulence
}

Zecong Qin, Hugues Faller, Bérengère Dubrulle, Aurore Naso, Wouter J.T. Bos

\section{To cite this version:}

Zecong Qin, Hugues Faller, Bérengère Dubrulle, Aurore Naso, Wouter J.T. Bos. Transition from nonswirling to swirling axisymmetric turbulence. Physical Review Fluids, 2020, 5 (6), 10.1103/PhysRevFluids.5.064602 . hal-02765845

\section{HAL Id: hal-02765845 \\ https://hal.science/hal-02765845}

Submitted on 4 Jun 2020

HAL is a multi-disciplinary open access archive for the deposit and dissemination of scientific research documents, whether they are published or not. The documents may come from teaching and research institutions in France or abroad, or from public or private research centers.
L'archive ouverte pluridisciplinaire HAL, est destinée au dépôt et à la diffusion de documents scientifiques de niveau recherche, publiés ou non, émanant des établissements d'enseignement et de recherche français ou étrangers, des laboratoires publics ou privés. 


\title{
Transition from non-swirling to swirling axisymmetric turbulence
}

\author{
Zecong Qin $\odot,{ }^{1}$ Hugues Faller $\odot,{ }^{2,3}$ Bérengère Dubrulle $\odot,{ }^{3}$ Aurore Naso $\odot,{ }^{1}$ \\ and Wouter J. T. Bos ${ }^{1}{ }^{1}$ \\ ${ }^{1}$ CNRS, Laboratoire de Mécanique des Fluides et d'Acoustique, UMR 5509, Univ Lyon, \\ Ecole Centrale de Lyon, INSA Lyon, Université Claude Bernard Lyon I, 69134, Écully, France \\ ${ }^{2}$ Université Paris-Saclay, CNRS, LIMSI, Laboratoire d'Informatique pour la Mécanique et les Sciences \\ de l'Ingénieur, 91400 Orsay, France \\ ${ }^{3}$ SPEC, CEA, CNRS, Université Paris-Saclay, CEA Saclay, 91191 Gif-sur-Yvette, France
}

(Received 17 October 2019; accepted 4 May 2020; published 3 June 2020)

\begin{abstract}
Strictly axisymmetric turbulence, i.e., turbulence governed by the Navier-Stokes equations modified such that the flow is invariant in the azimuthal direction, is a system intermediate between two- and three-dimensional turbulence. We investigate statistically steady states of this system by direct numerical simulation using a forcing protocol, which allows the injected energy in the toroidal and in the poloidal directions to be tuned independently. A sharp transition between a two-dimensional two-component (2D2C, nonswirling) flow and a two-dimensional three-component (2D3C, swirling) flow is observed. We derive a statistical model which reproduces this transition.
\end{abstract}

DOI: 10.1103/PhysRevFluids.5.064602

\section{INTRODUCTION}

In classical statistical physics terminology, transition refers to a change of a system state into another state when varying a control parameter. In phase transitions, the states are characterized by different symmetries, so that the transition reflects a spontaneous breaking of the symmetry. In such transitions, the order parameter, encoding the symmetry of the state, switches from 0 to a nonzero value, as a function of the control parameter. Such a type of transitions has also been observed in hydrodynamics, when the flow switches from the laminar to a turbulent state, as the different basic symmetries (translation, reflection, rotation, etc.) are gradually or abruptly broken, when increasing the Reynolds number. More recently, transitions between different turbulent states with different statistical symmetries were evidenced either experimentally [1,2] or numerically [3,4], still falling into the classical paradigm of spontaneous symmetry breaking.

In 1976 Frisch, Lesieur, and Sulem [5] investigated a new paradigm in turbulence by looking at transitions mediated by continuous changes of dimensionality of the flow from $D=2$ to $D=3$. In their investigation, the equivalent of the order parameter was the sign of the energy cascade direction, which changes from negative (backward cascade) to positive (forward cascade), for a value of $D$ close to 2 . Using the dimensionality as a control parameter is a rather abstract protocol that cannot be reproduced in natural flows. Instead, one can use body forces or geometrical confinement to introduce anisotropy in the flow and study the transition between two turbulent flows, with different effective dimensionality [6]. One can, for example, consider thin fluid layers [3,4], where the control parameter is the ratio between the thickness of the fluid layer and the scale at which energy is injected. One can also apply a magnetic field on a conductive fluid, resulting in the appearance of the Lorentz force, or the application of a solid body rotation, introducing the Coriolis force in the momentum balance [7]. In such cases, the control parameter is the ratio between the force and the inertia. When the control parameter becomes of order one, the flow becomes invariant, or almost, along the direction of the magnetic field or of the rotation axis, respectively. These 
two configurations therefore exhibit transitions from three-dimensional three-velocity-component (3D3C) to two-dimensional three-velocity-component (2D3C) turbulent flows. In other words, the flows in these examples have three nonzero velocity components, but these three components can vary either in the three dimensions or in a plane only (perpendicular to the axis of rotation or to the magnetic field).

In the present work, we investigate by direct numerical simulation a transition from $2 \mathrm{D} 2 \mathrm{C}$ to 2D3C turbulence, corresponding to a transition from strict 2D without vortex stretching to 2D3C (with vortex stretching). Such a transition occurs in strictly axisymmetric turbulence, an azimuthally invariant solution of the Navier-Stokes equations. This system is intermediate between 2D and 3D turbulence and was recently investigated by direct numerical simulation. It was shown in particular that an inverse energy cascade, responsible for the generation of large-scale coherent structures, and a direct helicity cascade towards small scales can coexist in this flow [8,9]. As predicted from theoretical works using statistical mechanics tools [10-12], different turbulent states were obtained for non-swirling (zero azimuthal velocity component, 2D2C) and for swirling flows (2D3C). We show here that a transition from the non-swirling to the swirling regime can be obtained by using the forcing anisotropy as a control parameter.

The paper is organized as follows. We first recall the definition of strictly axisymmetric turbulence and give some definitions in Sec. II. The numerical method and the results of the simulations carried out are presented in Sec. III. A statistical model is derived and its results are compared with these numerical data in Sec. IV. Finally, our conclusions are reported in Sec. V.

\section{AXISYMMETRIC TURBULENCE}

We consider incompressible, Newtonian, and isothermal axisymmetric turbulence. The flow is confined in a cylindrical domain of radius $R$ and of axis length $H$ and is a solution of the Navier-Stokes equations written in cylindrical coordinates $(r, \theta, z)$ for the three velocity components $\boldsymbol{u}=\left(u_{r}, u_{\theta}, u_{z}\right)$. The system is assumed invariant in the toroidal direction. In practice, this means that $\boldsymbol{u}=\left(u_{r}(r, z), u_{\theta}(r, z), u_{z}(r, z)\right)$ and that the pressure $p=p(r, z)$, so that any toroidal variation $\partial_{\theta}(\boldsymbol{u}, p)$ is zero. All external effects which can inject energy into the system in this simplified system are modeled by a forcing term added in the momentum equation. Under these assumptions, the flow is solution of the following system of equations:

$$
\begin{gathered}
D_{t}\left[u_{r}\right]=f_{r}+v \Delta_{r} u_{r}-\frac{1}{\rho} \partial_{r} p+\frac{u_{\theta}^{2}}{r}, \\
D_{t}\left[u_{z}\right]=f_{z}+v \Delta_{z} u_{z}-\frac{1}{\rho} \partial_{z} p, \\
D_{t}\left[u_{\theta}\right]=f_{\theta}+v \Delta_{\theta} u_{\theta}-\frac{u_{r} u_{\theta}}{r},
\end{gathered}
$$

where the operator $D_{t}$ is defined as

$$
D_{t}[g]=\partial_{t} g+u_{r} \partial_{r} g+u_{z} \partial_{z} g
$$

$\rho$ is the fluid density, $v$ its kinematic viscosity, and $\Delta_{i}$ are the three components of the cylindrical vector Laplacian. Incompressibility is ensured by the relation

$$
\frac{1}{r} \partial_{r}\left(r u_{r}\right)+\partial_{z} u_{z}=0
$$

The forcing $f_{i}$ considered in this investigation is based on the negative viscosity method [13] and will be defined more precisely later.

We will distinguish throughout this study the poloidal plane $(r, z)$ from the toroidal (azimuthal) direction. Note that axisymmetric turbulence can, in the absence of toroidal fluctuations, be reformulated, using a suitable change of variables, as purely Cartesian 2D turbulence [14,15], except for the exact form of the Laplacian. This means that to a good approximation axisymmetric 
turbulence with no swirl is effectively 2D. When a swirl is present, it can act as a source of toroidal vorticity (i.e., the vorticity associated with the poloidal movement), as can be checked by taking the curl of Eqs. (1) and (2). The vorticity is thus not conserved anymore, making the dynamics intermediate between 2D and 3D. A discussion of the dynamics of axisymmetric turbulence can be found in Ref. [10], where the invariants of the inviscid system and the evolution of the vorticity and angular momentum within this system are discussed.

The interaction between the toroidal and the poloidal dynamics is represented by the last terms on the right-hand sides of Eqs. (1) and (3). This coupling will be important in the following.

A measure of the flow "swirliness" is the ratio

$$
\gamma=\frac{E_{\mathrm{T}}}{E_{\mathrm{P}}}
$$

where $E_{\mathrm{T}}$ and $E_{\mathrm{P}}$, respectively, denote the toroidal and poloidal components of energy:

$$
\begin{gathered}
E_{\mathrm{T}}=\frac{\left\langle u_{\theta}^{2}\right\rangle}{2}, \\
E_{\mathrm{P}}=\frac{\left\langle u_{r}^{2}+u_{z}^{2}\right\rangle}{2},
\end{gathered}
$$

where $\langle\ldots\rangle$ are volume averages. If $\gamma=0$ the flow is purely poloidal $\left(u_{\theta}=0\right)$ and $2 \mathrm{D}$, whereas if $\gamma>0$ toroidal and poloidal velocity fluctuations coexist, and the flow is 2D3C [10]. These energy components are solutions of equations that can be derived from the Navier-Stokes equations (1)-(5) and formally write:

$$
\begin{aligned}
& \frac{d E_{\mathrm{P}}}{d t}=F_{\mathrm{P}}-\varepsilon_{\mathrm{P}}+\mathcal{T}, \\
& \frac{d E_{\mathrm{T}}}{d t}=F_{\mathrm{T}}-\varepsilon_{\mathrm{T}}-\mathcal{T},
\end{aligned}
$$

where $\varepsilon_{\mathrm{P}}$ and $\varepsilon_{\mathrm{T}}$ are viscous dissipation terms $\left(\varepsilon_{\mathrm{P}}, \varepsilon_{\mathrm{T}}>0\right), F_{\mathrm{P}}$ and $F_{\mathrm{T}}$ are terms corresponding to the work induced by the poloidal or the toroidal component of the forces $\left(F_{\mathrm{P}}, F_{\mathrm{T}}>0\right)$, and $\mathcal{T}$ is the transfer from the toroidal to the poloidal energy components:

$$
\mathcal{T}=\left\langle\frac{u_{\theta}^{2} u_{r}}{r}\right\rangle .
$$

A physical interpretation of this term is that, through conservation of angular momentum, inwarddirected (poloidal) flow increases the toroidal part of the kinetic energy. The detailed behavior of this term is important, since it is the only term which allows transfer of energy between the components.

In most of the following, we will consider time-averaged values during a statistically steady state. We therefore assume tacitly that all quantities are time averages, unless the time argument is explicitly mentioned [e.g., $\left.E_{\mathrm{T}}(t)\right]$.

\section{NUMERICAL SIMULATIONS}

\section{A. Method}

The Navier-Stokes equations (1)-(5) were integrated by using a fully spectral method based on an expansion of the velocity field in a basis consisting of a combination of Fourier modes in the axial direction and of Bessel functions in the radial one [8] (the code is based on the original design of Ref. [16]). In this approach, $n$ and $q$ are the mode numbers, respectively, associated to the axial and radial directions, $k_{n}$ being the axial wave number and $\gamma_{q}$ the $q$ th zero of the Bessel function $J_{1}$ in the radial direction. This allows us to define a global wave number defined as $k(n, q)=\left(k_{n}^{2}+\gamma_{q}^{2}\right)^{1 / 2}$.

As already mentioned, use was made of the negative viscosity method to force the flow [13]:

$$
\hat{f}_{i}(k)=c_{i} v h(k) \hat{u}_{i}(k), \quad i \in\{r, \theta, z\},
$$


where $\hat{g}$ denotes the spectral coefficient associated with a function $g(r, z), h(k)=k^{2}$ for $k_{f, \min } \leqslant$ $k \leqslant k_{f, \max }$ and zero elsewhere, and $c_{i}$ is a dimensionless forcing coefficient measuring the intensity of energy injection in the $i$ direction $(i \in\{r, \theta, z\})$. This forcing scheme allows solutions $u_{\theta}=0$, $u_{r}=0$ or $u_{z}=0$ for every value of the forcing strength. Contrary to the configuration investigated in Refs. [8,9], in which the flow was periodic in the axial direction, we imposed a confinement of the fluid in the cylindrical domain by using a suitable choice of the axial mode numbers $n$ [17].

We carried out 150 simulations in a cylindrical domain of radius $R=\pi$ and height $H=1.7 \pi$. In all of them the viscosity was set to $v=0.01$, and the same value is used in expression (12). The initial conditions of the simulations consist of random incompressible noise in all three velocity components with an initial value of the total energy of $E=0.01$. Calculations were performed using 52 modes in the radial direction and 45 modes in the axial one. The forcing band, $11 \leqslant k \leqslant 15$, corresponds to scales intermediate in size between the dissipation scale and the cylinder size, as in Ref. [9], which allows for the existence of cascades towards both small and large scales. The forcing coefficients were set such that $c_{r}=c_{z} \equiv c_{\mathrm{P}}$ and $c_{\theta} \equiv c_{\mathrm{T}}$.

\section{B. Parameters of the transition and their meaning}

The values of $c_{\mathrm{P}}$ and $c_{\mathrm{T}}$ were both chosen in the interval [1,3.6]. Statistically steady states were reached in all cases presented here. The results in the following subsection are obtained through statistics performed over the corresponding statistically steady states. In the sequel, we choose the order parameter to be the ratio $\gamma$ between the toroidal and poloidal energy components time averaged during the statistically steady state, as its variation from zero to nonzero values reveals the transition from 2D2C to 2D3C flow. We further checked that in the range of $c_{\mathrm{T}}$ and $c_{\mathrm{P}}$ values considered here, $\gamma$ depends, as a first approximation, only on the ratio of these parameters. We thus choose $c_{\mathrm{T}} / c_{\mathrm{P}}$ as the control parameter. Such a parameter physically represents the anisotropy of the forcing and reflects how large is the toroidal force term in the force balance compared with the poloidal force. The $\gamma$ parameter represents the response of the system to this forcing anisotropy, and which fraction of toroidal energy can be sustained in the flow, under such a constraint.

\section{Numerical results}

We first show in Fig. 1 the time evolution of the poloidal and toroidal energy components, for two distinct choices of the forcing parameters such that $c_{\mathrm{T}} / c_{\mathrm{P}}=1 / 3$ and $c_{\mathrm{T}} / c_{\mathrm{P}}=3$, respectively. Statistically steady states are observed after a transient time. In this regime, although both energy components fluctuate around finite values when $c_{\mathrm{T}} / c_{\mathrm{P}}=3$ [Fig. 1(b)], the toroidal energy component $E_{\mathrm{T}}$ is found to be zero for $c_{\mathrm{T}} / c_{\mathrm{P}}=1 / 3$ [Fig. 1(a)]. In that case, the flow therefore remains $2 \mathrm{D} 2 \mathrm{C}$, without swirl $\left(u_{\theta}=0\right)$.

In the following we report only on steady-state results. As mentioned all quantities indicate steady-state time averages. In Fig. 2(a) we show the steady-state values of $E_{\mathrm{P}}$ and $E_{\mathrm{T}}$ as a function of the forcing ratio. It is observed that the toroidal energy bifurcates from a zero to a nonzero value for a forcing ratio $\gamma$ somewhat above unity. At large values of $c_{\mathrm{T}} / c_{\mathrm{P}}, E_{\mathrm{T}}$ seems to saturate at a constant value, while the poloidal energy is reduced to a value below unity. There is considerable scatter in these values.

The scatter reduces when, instead of the energies, their ratio is considered. In Fig. 2(b), $\gamma$ is plotted as a function of $c_{\mathrm{T}} / c_{\mathrm{P}}$, and the bifurcation is here also clearly visible. A transition from a $2 \mathrm{D} 2 \mathrm{C}\left(E_{\mathrm{T}}=0\right)$ to a $2 \mathrm{D} 3 \mathrm{C}\left(E_{\mathrm{T}} \neq 0\right)$ state, occurring for $c_{\mathrm{T}} / c_{\mathrm{P}} \approx 1$, is observed. For weak toroidal forcing, the flow is purely poloidal, governed by purely $2 \mathrm{D}$ dynamics. When $c_{\mathrm{T}} / c_{\mathrm{P}} \approx 1$, a sharp transition is observed towards a swirling state, where both toroidal and poloidal velocity components are finite. An almost functional relation is observed between $\gamma$ and $c_{\mathrm{T}} / c_{\mathrm{P}}$.

The different natures of the flows for $c_{\mathrm{T}} / c_{\mathrm{P}}<1$ and $c_{\mathrm{T}} / c_{\mathrm{P}}>1$ are also illustrated in Fig. 3, in which the poloidal flow, time averaged during the stationary state is shown. Coherent large-scale structures reminiscent of $2 \mathrm{D}$ turbulence are clearly visible in the case $c_{\mathrm{T}} / c_{\mathrm{P}}<1$ [Fig. 3(a)], which 


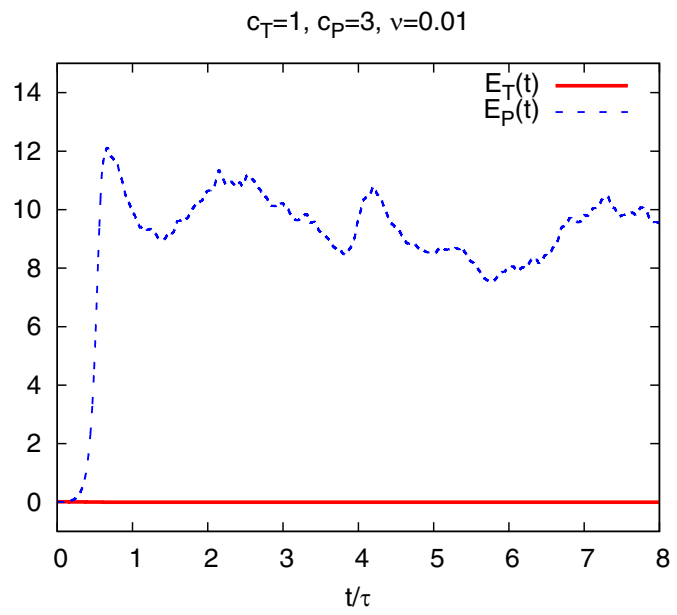

(a)

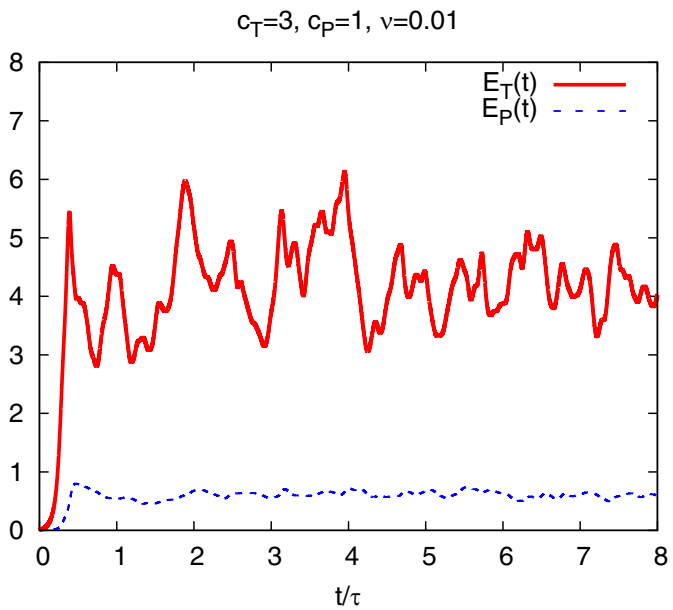

(b)

FIG. 1. Time evolution of the poloidal and toroidal energy components for: (a) $c_{\mathrm{P}}=3, c_{\mathrm{T}}=1\left(c_{\mathrm{T}} / c_{\mathrm{P}}=\right.$ $1 / 3)$ and (b) $c_{\mathrm{P}}=1, c_{\mathrm{T}}=3\left(c_{\mathrm{T}} / c_{\mathrm{P}}=3\right)$. Time is normalized by $\tau=R /(2 E / 3)^{1 / 2}$, where $E$ is the total kinetic energy of the flow, time averaged in the statistically steady state.

is not the case when $c_{\mathrm{T}} / c_{\mathrm{P}}>1$ [Fig. 3(b)], whatever the time interval chosen. The exact type of structures obtained in the former case may depend on the time interval chosen, as is well known in forced, 2D wall-bounded turbulence [18].

We illustrate the different dimensionalities of the flows for $c_{\mathrm{T}} / c_{\mathrm{P}}<1$ and $c_{\mathrm{T}} / c_{\mathrm{P}}>1$ by showing in Figs. 4(a)-4(c) energy spectra. For the definition of the energy spectra and the spectral fluxes we refer to Ref. [9]. When $c_{\mathrm{T}} / c_{\mathrm{P}}<1$ [Fig. 4(a)], a clear signature of an inverse energy cascade is observed, unlike in the case $c_{\mathrm{T}} / c_{\mathrm{P}}>1$ [Fig. 4(c)]. In this latter case for wave numbers smaller than the injection scale an approximate equipartition of energy is observed, with a wave number dependence roughly proportional to $k$. In the intermediate case Fig. 4(b), cascades in both directions seem to be present.

The cascade directions are confirmed in Figs. 4(d) and 4(f), in which the energy flux $\Pi(k)$ is shown. We observe in all cases, coexistence of "direct" and "inverse" cascade (with positive or 


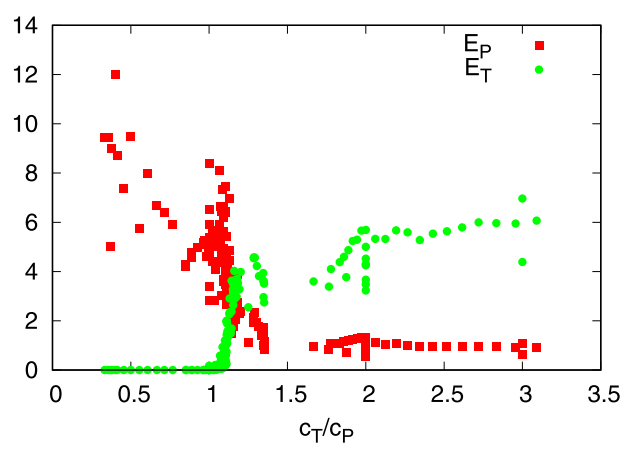

(a)

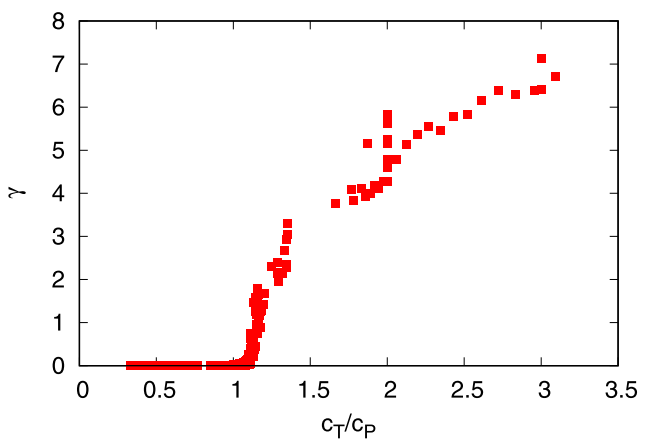

(b)

FIG. 2. (a) Steady-state values of the poloidal and toroidal energies as a function of $c_{\mathrm{T}} / c_{\mathrm{P}}$, for the complete set of simulations. (b) Ratio between the energy components time averaged during the steady state, $\gamma=E_{\mathrm{T}} / E_{\mathrm{P}}$, plotted as a function of the forcing parameter ratio.

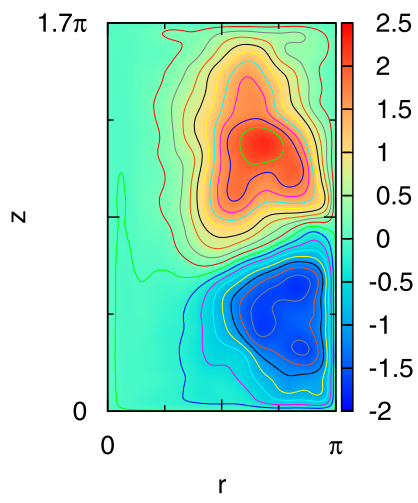

(a)

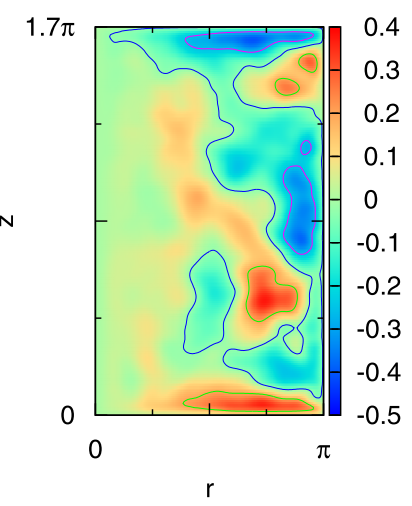

(b)

FIG. 3. Contour plots of the time-averaged poloidal stream function $\psi$ defined as $u_{r}=-\partial_{z} \psi / r, u_{z}=$ $\partial_{r} \psi / r$. The average is performed during the statistically steady state. The time interval over which $\psi$ is averaged is (a) $4<t / \tau<16$ and (b) $9<t / \tau<22$. Forcing parameters are (a) $c_{\mathrm{P}}=3, c_{\mathrm{T}}=1\left(c_{\mathrm{T}} / c_{\mathrm{P}}=1 / 3\right.$ ) and (b) $c_{\mathrm{P}}=1.2, c_{\mathrm{T}}=3.6\left(c_{\mathrm{T}} / c_{\mathrm{P}}=3\right)$. 


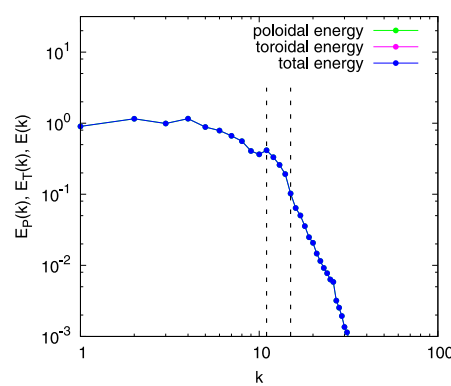

(a)

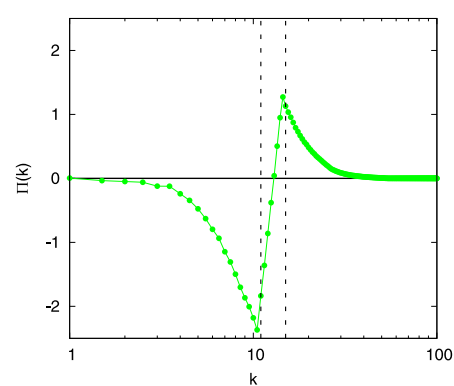

(d)

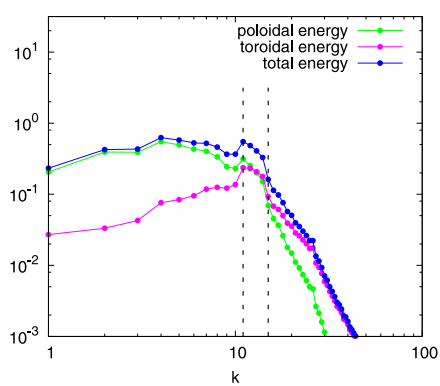

(b)

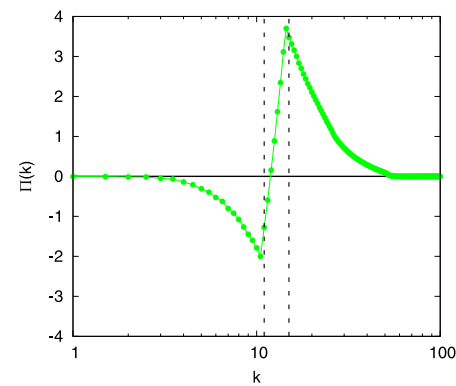

(e)

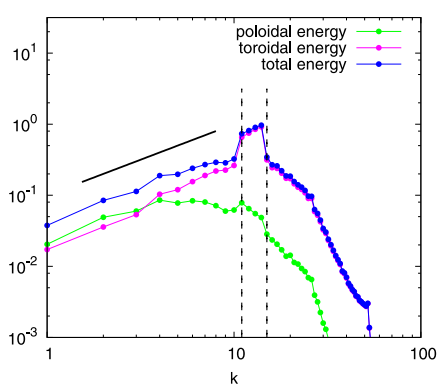

(c)

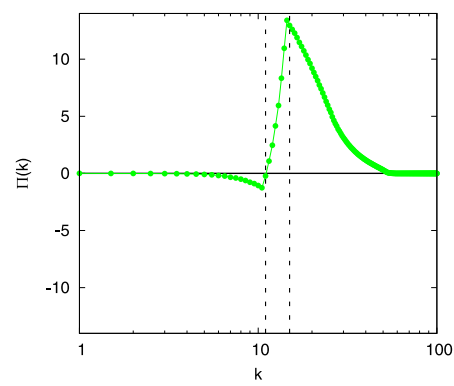

(f)

FIG. 4. Time-averaged (a, b, c) energy spectra of the total, poloidal and toroidal energy, and (d, e, f) energy flux. Values of the forcing parameters: (a,d) $c_{\mathrm{P}}=3, c_{\mathrm{T}}=1\left(c_{\mathrm{T}} / c_{\mathrm{P}}=1 / 3\right)$; (b, e) $c_{\mathrm{P}}=2.77, c_{\mathrm{T}}=3.13$ $\left(c_{\mathrm{T}} / c_{\mathrm{P}}=1.1\right) ;(\mathrm{c}, \mathrm{f}) c_{\mathrm{P}}=1.2, c_{\mathrm{T}}=3.6\left(c_{\mathrm{T}} / c_{\mathrm{P}}=3\right)$. The vertical dashed lines indicate the range of wave numbers in which forcing is applied. The solid black line in panel (c) indicates a scaling proportional to $k$.

negative flux), albeit with different relative intensity, resulting in different relative intensity for the poloidal to toroidal energy. For the purely poloidal flow [Fig. 4(d)], an important part of the energy is transferred to lower wave numbers. This is not the case in the swirling regime [Fig. 4(f)], where the energy flux is predominantly directed towards the large wave numbers. In the intermediate case [Fig. 4(b)] fluxes in both directions are clearly present. Such behavior is reminiscent of the "split energy cascade" scenario, also observed for geometrically constrained 3D flow, as the aspect ratio (the control parameter) is varied from 0 to 1 [6].

In Figs. 5 and 6 we document all terms on the right-hand side of Eqs. (9) and (10). Insight in the behavior of these terms will allow us to model and thereby better understand the dynamics of the system. We observe in Fig. 5(a) that the dissipation displays a bifurcation similar to that exhibited by the energy. The poloidal and toroidal dissipations behave qualitatively as the energy components. The energy forcing terms $F_{\mathrm{P}}$ and $F_{\mathrm{T}}$ are plotted as a function of the poloidal and toroidal components of the energy, respectively, in Fig. 5(b). It is shown that their dependence on the associated energy components is not so far from a linear relation.

The transfer $\mathcal{T}$ is perhaps the most intriguing contribution of the balance equations, since it couples the energy of the two components. It is shown in Fig. 6 that this quantity can have both signs depending on the value of the control parameter. For $c_{\mathrm{T}} / c_{\mathrm{P}}<1$ (2D2C turbulence), no transfer occurs between both flow components, as expected. For $1<c_{\mathrm{T}} / c_{\mathrm{P}} \lesssim 1.3$, energy is preferentially transferred from the poloidal to the toroidal field $(\mathcal{T}<0$, the poloidal field "feeds" the toroidal one), whereas for higher values of $c_{\mathrm{T}} / c_{\mathrm{P}}$ it is transferred from the toroidal to the poloidal directions $(\mathcal{T}>0)$. In Fig. 6(b), where the transfer is plotted as a function of $\gamma$, it is observed that this change of sign takes place for a value of $\gamma \approx 2$.

To summarize the observations, we have shown numerically (consistently with Figs. 6 and 2) the existence of a transition from $2 \mathrm{D} 2 \mathrm{C}$ to $2 \mathrm{D} 3 \mathrm{C}$ turbulent flows. The control parameter of this transition 


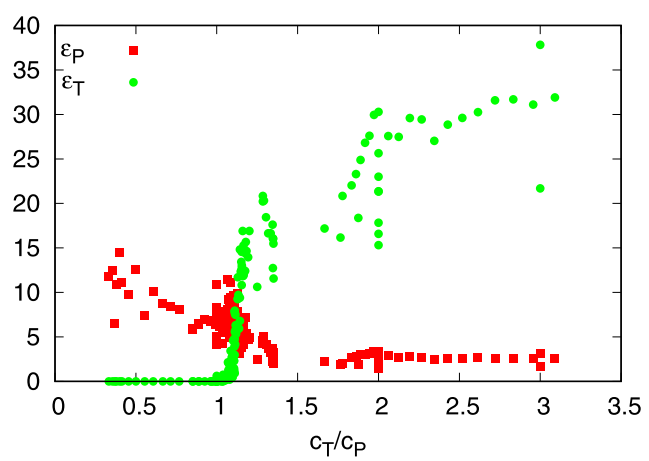

(a)

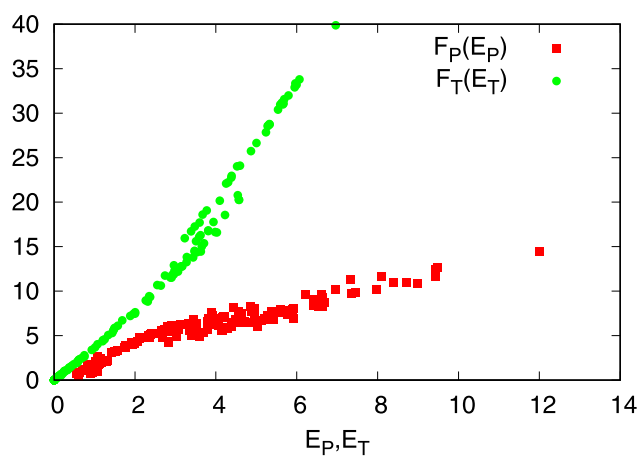

(b)

FIG. 5. (a) Steady-state values of the poloidal and toroidal dissipation rates as a function of the forcing ratio $c_{\mathrm{T}} / c_{\mathrm{P}}$, for the complete set of simulations. (b) Value of the energy injection rates $F_{\mathrm{P}}, F_{\mathrm{T}}$, plotted as a function of the energies $E_{\mathrm{P}}, E_{\mathrm{T}}$.

is the ratio between the forcing coefficients, $c_{\mathrm{T}} / c_{\mathrm{P}}$, the transition occurring for $c_{\mathrm{T}} / c_{\mathrm{P}} \approx 1$. The order parameter is the ratio between the toroidal and poloidal components of energy, $\gamma$, which measures the flow "componentiality": 2D2C for $\gamma=0,2 \mathrm{D} 3 \mathrm{C}$ for $\gamma \neq 0$. We can thereby distinguish three characteristic states:

(1) $\mathcal{T}=0 ; \gamma=0$; the spectral energy flux is mainly negative, resulting in an inverse cascade of poloidal energy, making the dynamics effectively $2 \mathrm{D}$

(2) $\mathcal{T}<0 ; \gamma<2$; the poloidal flow dominates; there is an inverse cascade at large scale coexisting with a small-scale direct cascade of comparable intensity

(3) $\mathcal{T}>0 ; \gamma>2$; the toroidal energy dominates; there is mainly a small-scale direct cascade; the dynamics is therefore effectively $3 \mathrm{D}$.

In the following we will derive a model aiming to reproduce this transition.

\section{STATISTICAL MODEL}

In order to better understand the transition, we developed a statistical model of the dynamics. Our aim was to derive the simplest possible model able to capture the steady states of the system (9) and (10), by estimating the five terms appearing on the right-hand sides of these equations. These terms are the forcing terms $F_{\mathrm{P}}, F_{\mathrm{T}}$, dissipation terms $\varepsilon_{\mathrm{P}}, \varepsilon_{\mathrm{T}}$, and transfer $\mathcal{T}$. We will model these terms using rough estimates inspired by turbulence models. The goal is not an exact reproduction of the results, but the determination of the main physical ingredients to reproduce the observations. 


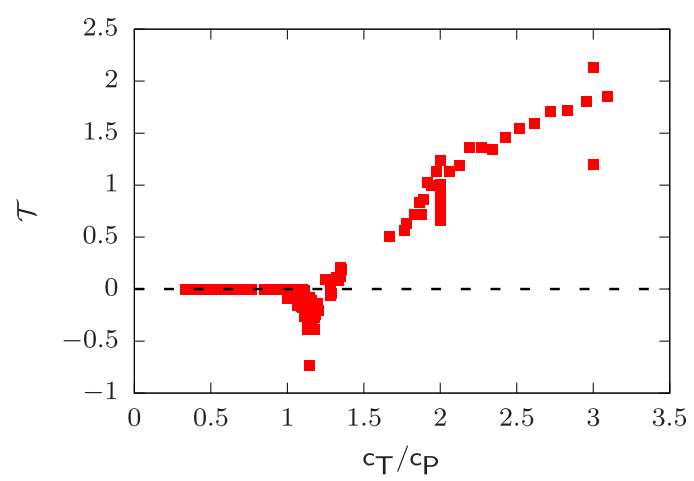

(a)

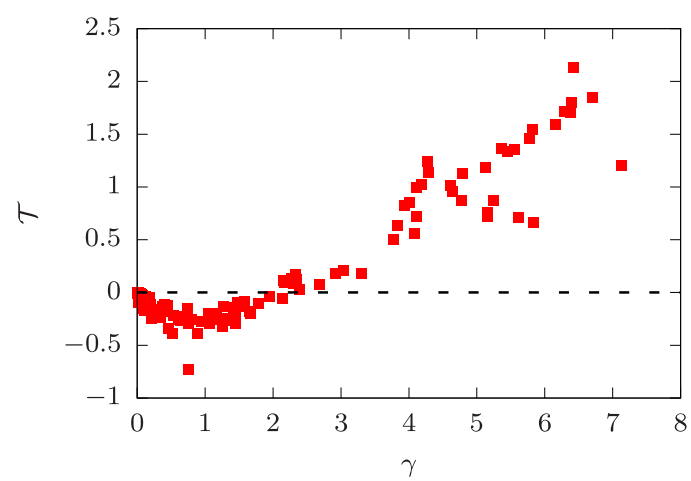

(b)

FIG. 6. Transfer from the toroidal to the poloidal energy components, $\mathcal{T}=\overline{\left\langle u_{\theta}^{2} u_{r} / r\right\rangle}$, plotted as a function of (a) $c_{\mathrm{T}} / c_{\mathrm{P}}$ and (b) $\gamma$.

However, we will show that the model is precise enough to quantitatively compare the main quantities to the direct numerical simulations.

The forcing terms correspond to a linear forcing at a given scale. The simplest possible model to represent this as a function of global quantities is a linear forcing at all scales,

$$
F_{\mathrm{P}}=C_{\mathrm{P}} E_{\mathrm{P}}, \quad F_{\mathrm{T}}=C_{\mathrm{T}} E_{\mathrm{T}} .
$$

The main simplification is here that the forcing is chosen proportional to the total energy instead of only the energy in a spectral band. This assumption roughly holds for most of the data, as observed in Fig. 5.

The dissipation terms are modeled using Taylor's dissipation rate expression [19],

$$
\begin{aligned}
\varepsilon_{\mathrm{P}} & =d_{\mathrm{P}} \frac{E_{\mathrm{P}}}{\tau}, \\
\varepsilon_{\mathrm{T}} & =d_{\mathrm{T}} \frac{E_{\mathrm{T}}}{\tau},
\end{aligned}
$$

where $d_{\mathrm{P}}$ and $d_{\mathrm{T}}$ are dimensionless model parameters, and $\tau$ is a typical timescale associated with the poloidal motion:

$$
\tau \sim L / E_{\mathrm{P}}^{1 / 2}
$$

In this expression $L$ is a length scale. 
The transfer of energy between poloidal and toroidal components is modeled as

$$
\mathcal{T}=\alpha \tau \frac{E_{\mathrm{T}} E_{\mathrm{P}}}{L^{2}}(\gamma-2),
$$

where $\alpha$ is a dimensionless parameter of the model and $\tau$ can be estimated as in Eq. (16). The shape of this model is inspired by the associated transfer term in a classical Reynolds-stress model formulated in cylindrical coordinates [20]. The value 2 in brackets is chosen since the transfer is observed to have a zero crossing around $\gamma=2$, as observed in Fig. 6(b).

Using these expressions, the model writes

$$
\begin{gathered}
\frac{d E_{\mathrm{P}}}{d t}=C_{\mathrm{P}} E_{\mathrm{P}}-d_{\mathrm{P}} \frac{E_{\mathrm{P}}^{3 / 2}}{L}+\alpha \frac{E_{\mathrm{T}} E_{\mathrm{P}}^{1 / 2}}{L}(\gamma-2), \\
\frac{d E_{\mathrm{T}}}{d t}=C_{\mathrm{T}} E_{\mathrm{T}}-d_{\mathrm{T}} \frac{E_{\mathrm{T}} E_{\mathrm{P}}^{1 / 2}}{L}-\alpha \frac{E_{\mathrm{T}} E_{\mathrm{P}}^{1 / 2}}{L}(\gamma-2) .
\end{gathered}
$$

More details on the derivation and modeling assumptions can be found in Ref. [17].

Now, to simplify the algebra, we introduce the quantity

$$
\zeta=\frac{c_{\mathrm{T}}}{c_{\mathrm{P}}}
$$

Steady states of the system (18) and (19) can be readily determined. They are solutions of the following equation for $\gamma$ :

$$
\gamma\left[\gamma^{2}+\frac{1-2 \zeta}{\zeta} \gamma+\frac{d_{\mathrm{T}}-d_{\mathrm{P}} \zeta-2 \alpha}{\alpha \zeta}\right]=0,
$$

with solution $\gamma=0$ or

$$
\gamma_{ \pm}=\left(1-\frac{1}{2 \zeta}\right) \pm \sqrt{1-\frac{d_{\mathrm{T}}}{\alpha \zeta}+\frac{1}{\zeta}+\frac{1}{4 \zeta^{2}}+\frac{d_{\mathrm{P}}}{\alpha}}
$$

We observe immediately that this solution only depends on $\zeta$ and not on the individual values of the forcing parameters, as in the simulations. Furthermore, we see that the length scale $L$ does not intervene in the expression. The solution depends thus on two parameters, $d_{\mathrm{T}} / \alpha$ and $d_{\mathrm{P}} / \alpha$. Stable solutions are $\gamma=0$ and $\gamma_{+}$. We have fitted this last solution to the data of $\gamma(\zeta)$ for $\gamma>1$ and found the values $d_{\mathrm{P}} / \alpha=38.4 \pm 1$ and $d_{\mathrm{T}} / \alpha=46.1 \pm 1$. This solution is plotted in Fig. 7(a). It is observed that some rough agreement is observed. Indeed, the model, like the data, describes a transition from $\gamma=0$ to a $\gamma \neq 0$. Furthermore, the model yields a functional relation between $\gamma$ and $\zeta$ for most values of $\zeta$ and a saturation to a finite value for large values of $\zeta$. For the model this saturation is given by the value $\gamma=1+\sqrt{1+d_{\mathrm{P}} / \alpha}$. From this expression it is clear that the transfer is essential to prevent the toroidal energy from diverging to infinity. Indeed, Eq. (19) shows that all terms are linear functions of $E_{\mathrm{T}}$ except the transfer term, so that in the absence of this term no nonlinear saturation mechanism is present for the toroidal energy.

There is, however, also some disagreement. The model predicts two nontrivial branches beyond the bifurcation, suggesting a subcritical transition with hysteresis. Such behavior with a subcritical transition between two different turbulent states was recently also observed in a different system, thin-layer rotating Rayleigh-Bénard convection [21]. The model prediction of subcriticality in the present case is in particular visible in the zoom in Fig. 7(b). We have not detected any hysteresis in our simulations, but it should be mentioned here that the interval in which the model predicts hysteresis is very narrow, $1.14<\zeta<1.15$. Furthermore, in this interval, where $E_{\mathrm{T}}$ is very small, the direct viscous effect on the toroidal dissipation is presumably large, so that the high Reynolds numbers estimate (15) is inaccurate here. Refining the model to take this into account would introduce Reynolds number effects into the description and $\gamma$ would not uniquely depend on $\zeta$ anymore. This complexification of the model is not attempted here. 


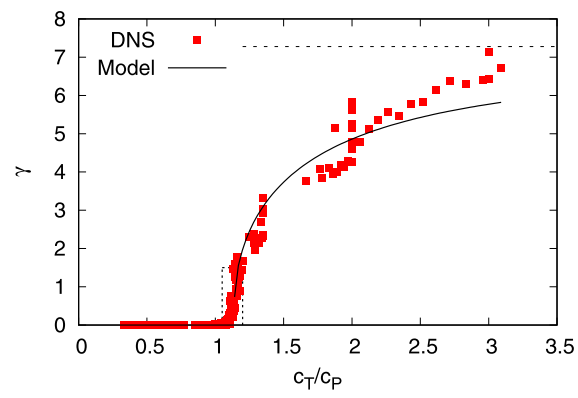

(a)

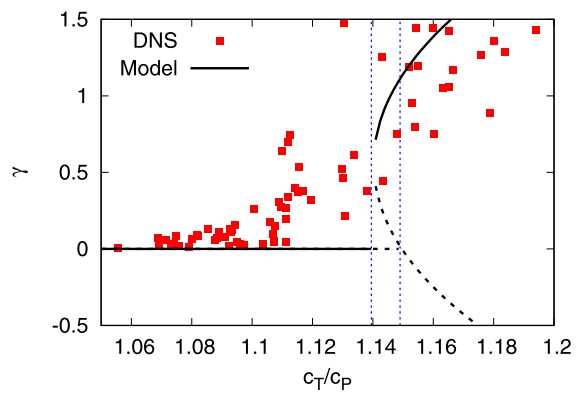

(b)

FIG. 7. (a) Comparison of the model solution (22) to the data, for an optimal fit of the constants. Also shown is the asymptotic value obtained in the model for large values of $\zeta$ (dashed line). The dashed box is zoomed upon in panel (b). Here we see that the interval in which hysteresis is predicted by the model is very narrow (vertical dashed lines). This hysteresis is not observed in the simulations. The dashed black line represents the unstable branch of the solution of the model.

\section{DISCUSSION}

Direct numerical simulations of axisymmetric turbulence forced by a negative viscosity method show the existence of a sharp transition between 2D2C, purely poloidal, or non-swirling turbulence, and 2D3C poloidal-toroidal, or swirling turbulence. Despite its obvious geometrical limitations (the axisymmetric assumption), our results can be used for a qualitative understanding of several puzzling empirical facts of a real laboratory flow, the von Kármán flow. In such a system, the turbulence is achieved through counterrotation of two impellers with blades of given curvature. In Ref. [22] the ratio of the toroidal to poloidal kinetic energy has been studied as a function of the curvature, and a simple quasilinear law was found. This can be understood by the fact that the curvature influences the ratio of the toroidal to poloidal injected energy, and plays a similar role as $c_{\mathrm{T}} / c_{\mathrm{P}}$. For high enough Reynolds number, when the curvature exceeds a critical value, a symmetry-breaking bifurcation is further seen in the von Kármán system, between a state where the mean turbulent flow has a zero average angular momentum and two-cell topology for the poloidal component like in Fig. 3(a), and a state where the mean turbulent flow has nonzero average angular momentum with a one cell topology for the poloidal component, reminiscent of Fig. 3(b). It would therefore be interesting to generalize our statistical model to account for nonaxisymmetric fluctuations to try and fit the experimental bifurcation.

Conversely, it would be interesting to investigate numerically the dependence of the transition on the Reynolds number and see whether a critical Reynolds number exists, under which the transition does not happen anymore, thereby mimicking the experimental behavior. In case this is true, a basic 
open question is to derive a new closure since our model is derived using assumptions usually valid in the limit of high Reynolds number turbulence. Finally, a transition from 2D3C (strictly axisymmetric) to 3D3C turbulence could be investigated using similar tools.

\section{ACKNOWLEDGMENTS}

Shuojun Li is gratefully acknowledged for providing his code for 2D turbulence. Z.Q. was supported by a grant from the China Scholarship Council. All simulations were carried out using the facilities of the PMCS2I (École Centrale de Lyon). B.D. acknowledges funding from ANR EXPLOIT, grant agreement ANR-16-CE06-0006-01.

[1] F. Ravelet, L. Marié, A. Chiffaudel, and F. Daviaud, Multistability and Memory Effect in a Highly Turbulent Flow: Experimental Evidence for a Global Bifurcation, Phys. Rev. Lett. 93, 164501 (2004).

[2] Y.-C. Xie, G.-Y. Ding, and K.-Q. Xia, Flow Topology Transition Via Global Bifurcation in Thermally Driven Turbulence, Phys. Rev. Lett. 120, 214501 (2018).

[3] A. Celani, S. Musacchio, and D. Vincenzi, Turbulence in More Than Two and Less Than Three Dimensions, Phys. Rev. Lett. 104, 184506 (2010).

[4] S. J. Benavides and A. Alexakis, Critical transitions in thin layer turbulence, J. Fluid Mech. 822, 364 (2017).

[5] U. Frisch, M. Lesieur, and P. Sulem, Crossover Dimensions for fully Developed Turbulence, Phys. Rev. Lett. 37, 1312 (1976).

[6] A. Alexakis and L. Biferale, Cascades and transitions in turbulent flows, Phys. Rep. 767-769, 1 (2018).

[7] P. A. Davidson, Turbulence in Rotating, Stratified and Electrically Conducting Fluids (Cambridge University Press, Cambridge, 2013).

[8] B. Qu, W. J. T. Bos, and A. Naso, Direct numerical simulation of axisymmetric turbulence, Phys. Rev. Fluids 2, 094608 (2017).

[9] B. Qu, A. Naso, and W. J. T. Bos, Cascades of energy and helicity in axisymmetric turbulence, Phys. Rev. Fluids 3, 014607 (2018).

[10] N. Leprovost, B. Dubrulle, and P.-H. Chavanis, Dynamics and thermodynamics of axisymmetric flows: Theory, Phys. Rev. E 73, 046308 (2006).

[11] A. Naso, R. Monchaux, P. H. Chavanis, and B. Dubrulle, Statistical mechanics of Beltrami flows in axisymmetric geometry: Theory reexamined, Phys. Rev. E 81, 066318 (2010).

[12] A. Naso, S. Thalabard, G. Collette, P.-H. Chavanis, and B. Dubrulle, Statistical mechanics of Beltrami flows in axisymmetric geometry: Equilibria and bifurcations, J. Stat. Mech. (2010) P06019.

[13] Y. Kaneda, T. Ishihara, M. Yokokawa, K. I. Itakura, and A. Uno, Energy dissipation rate and energy spectrum in high resolution direct numerical simulations of turbulence in a periodic box, Phys. Fluids 15, L21 (2003).

[14] K. Mohseni, Statistical equilibrium theory for axisymmetric flows: Kelvin's variational principle and an explanation for the vortex ring pinch-off process, Phys. Fluids 13, 1924 (2001).

[15] C. C. Lim, Coherent structures in an energy-enstrophy theory for axisymmetric flows, Phys. Fluids 15, 478 (2003).

[16] S. Li, D. Montgomery, and W. Jones, Two-dimensional turbulence with rigid walls, Theor. Comput. Fluid Dyn. 9, 167 (1997).

[17] Z. Qin, Transitions in axisymmetric turbulence, Ph.D. thesis, Université de Lyon (2019).

[18] G. van Heijst, H. Clercx, and D. Molenaar, The effects of solid boundaries on confined two-dimensional turbulence, J. Fluid Mech. 554, 411 (2006).

[19] G. Taylor, Statistical theory of turbulence, Proc. R. Soc. London A 151, 421 (1935). 
[20] B. Launder and A. Morse, Numerical prediction of axisymmetric free shear flows with a Reynolds-stress closure, in Turbulent Shear Flows I (Springer, New York, 1979), pp. 279-294.

[21] B. Favier, C. Guervilly, and E. Knobloch, Subcritical turbulent condensate in rapidly rotating RayleighBénard convection, J. Fluid Mech. 864, R1 (2019).

[22] F. Ravelet, Bifurcations globales hydrodynamiques et magnétohydrodynamiques dans un écoulement de von Kármán turbulent, Ph.D. thesis, École Polytechnique, Palaiseau (2005). 